\title{
RM18
}

\section{Hybrid Geostatistics: Object-based Simulations Using MPS-generated Meandering Channels}

\author{
P. Renard* (University of Neuchatel), G. Mariethoz (University of New \\ South Wales), A. Comunian (University of New South Wales) \& J. \\ Straubhaar (University of Neuchatel)
}

\section{SUMMARY}

Object-based simulation methods are some of the most widely used approaches for modeling geological reservoirs and quantifying geological uncertainty. However, one major drawback of the object-based approach is its lack of flexibility. The objects are often defined with basic analytical shapes, and as such they may not reflect the complexity of the actual geobodies present in the subsurface. This results in models that lack realism, as well as difficulties in conditioning especially in the presence of dense datasets. To tackle these issues, we propose to stochastically generate objects using an analog-based approach. Instead of defining objects by a limited number of parameters, objects are produced by using a multiplepoint geostatistics (MPS) algorithm, which is a non-parametric process. Each new object is then drawn from an ensemble of possible members, all presenting similar statistical properties than a given reference object, namely a training image. 


\section{Introduction}

Object-based simulation methods are some of the most widely used approaches for modeling geological reservoirs and quantifying geological uncertainty. The general principle is to randomly place predefined shapes (objects) in the modeled volume. The objects shape, properties and spatial density can be parameterized to account for geological interpretation or various types of data, in particular soft information.

However, one major drawback of the object-based approach is its lack of flexibility. The objects are often defined with basic analytical shapes (e.g. sinusoids for channels, 3D ellipsoids for lenses, 2D ellipses for fractures), and as such they may not reflect the complexity of the actual geobodies present in the subsurface. This results in models that lack realism, as well as difficulties in conditioning especially in the presence of dense datasets.

To tackle these issues, we propose to stochastically generate objects using an analog-based approach. Instead of defining objects by a limited number of parameters (i.e. centroid, sinuosity, aspect ratio, etc), objects are produced by using a multiple-point geostatistics (MPS) algorithm, which is a nonparametric process. Each new object is then drawn from an ensemble of possible members, all presenting similar statistical properties than a given reference object, namely a training image.

\section{Methodology}

Our approach consists in using MPS within the object-based simulator to generate one object at the time. It is illustrated with the simulation of channelized sand bodies in a low-permeability matrix, which is typically a complex reservoir setting because of the large-scale connected structures that are difficult to represent using MPS, as well as the complex shapes of the meandering channels objects, which is a challenge for object-based methods.

The MPS simulation is handled differently than in the classical approach where the training image is an explicit representation of stationary spatial patterns. Instead, the training image is made of a single object (in this case a present-day meandering river channel), which is transformed such that it can be represented as a stationary $1 \mathrm{D}$ process (Oliver 2002). This training image is then used to generate new stochastic objects that have similar morphological properties as the object used as training image.

One advantage is that each object can be easily conditioned to data (hard and soft), because the MPS approach allows for increased flexibility (Mariethoz et al. 2014). Moreover, there is more variability in the generated objects compared to the usual parametric shapes, which allows conveying more geological realism to the models.

\section{Conclusions}

Traditionally, object-based simulation has been criticised for its lack of flexibility while MPS was challenged by large-scale connectivity reproduction. The methodology presented is a hybrid between object-based simulation and multiple-point geostatistics, borrowing the best of both worlds: it allows producing large connected structures due to its object-based nature, and at the same time the conditioning capabilities of MPS allow dealing with diverse types of data. 


\section{EAGE}

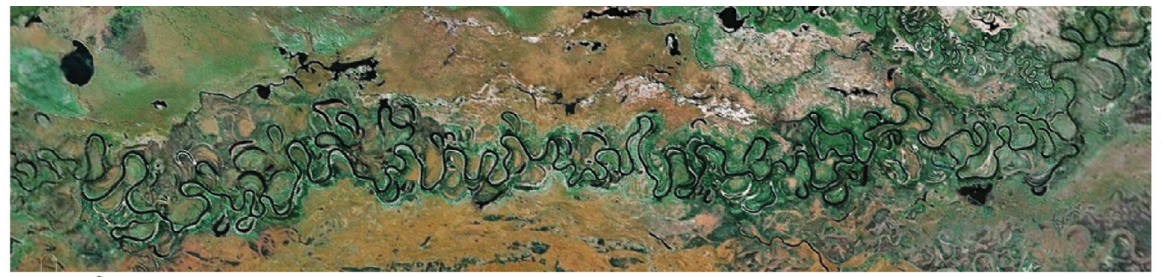

Training Image

Birch Creek, Alaska, USA

$612069 \mathrm{E} / 7320486 \mathrm{~N}$

तथ

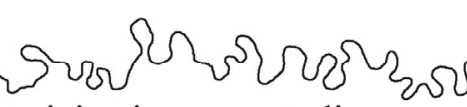

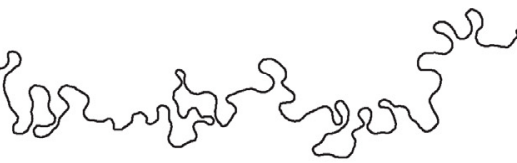

$\longmapsto 5 \mathrm{~km}$

Digitized training image centerline

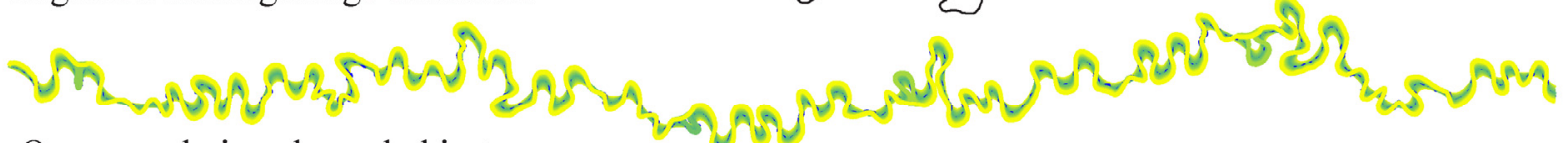

One meandering channel object

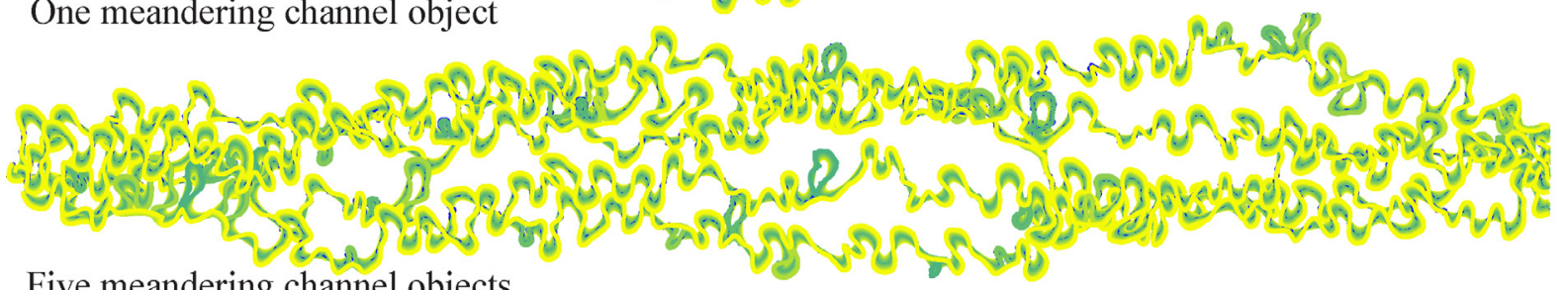

Five meandering channel objects

Figure 1 Top: representation of the type of objects desired, in this case a digitized meandering river channel. The object is analysed and transformed in a $1 D$ representation which can be used as training image. The training image is then the base to generate new meandering river channels objects that have similar morphological properties as the original digitized object.

\section{References}

Oliver, D. [2002] Conditioning Channel Meanders to Well Observations. Math. Geol., 34, 185-201.

Mariethoz, G., Comunian, A., Irarrazaval, I. and Renard, P. [2014] Analog-based meandering river simulation. Water Resources Research, 50(2). 